\title{
Dose Escalation
}

National Cancer Institute

\section{Source}

National Cancer Institute. Dose Escalation. NCI Thesaurus. Code C107213.

A stepwise increase in the amount of a therapeutic or investigational agent given to an individual. 